Research Paper

\title{
A dual-specific IGF-I/II human engineered antibody domain inhibits IGF signaling in breast cancer cells
}

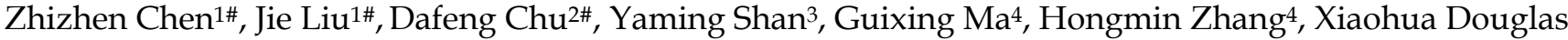 \\ Zhang ${ }^{1}$, Pu Wang 5 , Qiang Chen ${ }^{1}$, Chuxia Deng1, Weizao Chen ${ }^{6}$, Dimiter S. Dimitrov7, Qi Zhao ${ }^{\circledR}$ \\ 1. Faculty of Health Sciences, University of Macau, Macau, China. \\ 2. Department of Bioengineering, University of California, Los Angeles, California, USA. \\ 3. National Engineering Laboratory for AIDS Vaccine, School of Life Science, Jilin University, Changchun, Jilin, China; \\ 4. Department of Biology, Guangdong Provincial Key Laboratory of Cell Microenvironment and Disease Research, Shenzhen Key Laboratory of Cell \\ Microenvironment, SUSTech-HKU joint laboratories for matrix biology and diseases, Southern University of Science and Technology, Shenzhen, \\ Guangdong, China. \\ 5. Shenzhen Institutes of Advanced Technology, Chinese Academy of Sciences, Guangdong, China. \\ 6. Center for Cancer Research, National Cancer Institute-Frederick, National Institutes of Health, Maryland, USA. \\ 7. Center for Antibody Therapeutics, University of Pittsburgh Medical School, Pennsylvania, USA. \\ "equal contribution \\ $\square$ Corresponding author: Qi Zhao, PhD, E12-3022, University of Macau, Taipa, Macau, China. Email: zhaoqi@alumni.cuhk.net; qizhao@umac.mo; Tel: \\ $+853-88224824$
}

(c) Ivyspring International Publisher. This is an open access article distributed under the terms of the Creative Commons Attribution (CC BY-NC) license (https://creativecommons.org/licenses/by-nc/4.0/). See http://ivyspring.com/terms for full terms and conditions.

Received: 2018.03.07; Accepted: 2018.04.10; Published: 2018.05.21

\begin{abstract}
The insulin-like growth factors (IGFs), IGF-I and IGF-II, are essential for regulating cell growth, differentiation and metastasis of a broad range of malignancies. The IGF-I/II actions are mediated through the IGF receptor type 1 (IGF-IR) and the insulin receptor (IR), which are overexpressed in multiple types of tumors. Here, we have firstly identified a human engineered antibody domain (eAd) from a phage-displayed VH library. The eAd suppressed the signal transduction of IGF-IR mediated by exogenous IGF-I or IGF-II in breast cancer cell lines through neutralizing both IGF-I and IGF-II. It also significantly inhibited the growth of breast cancer cells. Therefore, the anti-IGF-I/II eAd offers an alternative approach to target both the IGF-IR signaling pathways through the inhibition of IGF-I/II.
\end{abstract}

Key words: Insulin-like growth factor, Yeast display, Affinity maturation, eAd

\section{Introduction}

Insulin-like growth factor (IGF) dysregulation has been implicated in the development and progression of many tumor types. The IGF system is composed of IGF-I and IGF-II, IGF receptor type 1 (IGF-1R), IGF receptor type 2 (IGF-2R), insulin receptor (IR), and six high-affinity IGF binding proteins (IGFBP) [1]. The interaction between IGF1R and its ligands is involved in a variety of physiologic processes, including immunity as well as in diseases including infections and cancers. Numerous studies demonstrate the importance of IGF-1R and its ligands for tumor survival in a broad range of human tumors, including breast, ovarian, and pediatric cancers $[1,2]$. IGF-I and IGF-II have been proven to play important roles in neoplasm [3]. Most of the circulating IGF-I and IGF-II are associated with IGFBPs [4]. The free ligand concentration is too low to interact with cell surface receptors. Upon activation by the mitogenic ligands IGF-I and IGF-II, the IGF-1R is activated to trigger intracellular signaling pathways (Ras/MEK/ ERK and PI3K/AKT pathways) that result in tumor progression and metastasis [1]. Because the IGF-1R and IR have $70 \%$ amino acid identity and $84 \%$ identity within the catalytic domain, they form heterodimeric receptors. Although human IGF-I and IGF-II have a high degree of sequence similarity, the binding sites for IGF-I and IGF-II on receptors are distinct. IGF-II binds the IR isoform $\mathrm{A}$ and the hybrid receptor 
IGF-1R/IR, whereas IGF-I bind to IR or IGF-1R/IR with low affinity (non-physiological levels) [4]. The overexpression of IR in primary malignant tumors is correlated with tumor survival [5]. Activation of the IR by IGF-II has been shown to be the predominant IGF signaling receptor in breast cancer [6].

The modulation of the activity of the IGF axis could be an approach to anticancer therapy [7]. Although many antibodies and inhibitors targeting IGF-1R have been developed and entered early clinical trials, therapy against IGF-1R seems to partially inhibit the IGF-mediating signal transduction [8]. IGF-II-mediated signaling through IR-A results in tumor resistance during treatment with IGF$1 \mathrm{R}$ targeting [9]. Thereby, most anti-IGF-1R inhibitors fail to abolish IGF signaling because they incompletely block the action of IGF-II on the IR-A homodimers [10]. Although both IGF-1R and IR can be targeted, glucose homeostasis can be severely dysregulated. Hyperglycemia might appear in patient [11].

Elevated levels of IGF-I and/or IGF-II are observed in tumors such as neuroblastoma, Ewing sarcoma, and osteosarcoma [12]. Like IGF-I, IGF-II may be secreted by stromal cells [13]. Within the tumor microenvironment, both IGF-I and IGF-II facilitate disease progression through autocrine or paracrine interactions between stromal and tumor cells [2]. In addition, IGFs play role in endocrine signaling on adjacent epithelial cells when they are circulated in the vascular system. In recent antibodies targeting IGFs become intense in preclinical and clinical research [14-17]. We previously reported an anti-IGF-I/II monoclonal antibody (mAb) (m708.5) [18] and an anti-IGF-II mAb (m610) [19]. They are proposed to eliminate the efflux of IGFs from tumor tissues and circulating IGFs in the serum. The $\mathrm{mAb}$ m610 blocked the growth/ of human breast cancer lines in vitro and the migration of prostate cancer cells implanted in human adult bones [20]. The mAb m708.5 inhibited IGF signaling through both the IGF-1R and IR-A pathways and the in vivo growth of pediatric tumors [16]. Although full-size IgGs (150 $\mathrm{kDa}$ ) are favoured by their long half-life, tumor penetration by IgGs, in some cases, is limited due to their large size and uptake by $\mathrm{Fc}_{\mathrm{c}}$ receptors on reticulo-endothelial cells [21, 22]. Engineered antibody domains (eAds) with unique properties are a novel class of candidate therapeutics for cancer [23] and infectious diseases [24]. The eAds may access the sites that are not accessible to full-size antibodies [25, 26].

In the present study, we describe the identification and characterization of an IGF-I/ II-neutralizing eAd s7g1 derived from a human variable fragment of heavy chain $(\mathrm{VH})$. It significantly blocked the signal transduction mediated by the interaction of IGF-I or IGF-II with IGF-1R and IGF-II with the insulin receptor. It also inhibited the growth of breast cancer cells. These results suggest that s7g1 may have potential as a candidate therapeutic.

\section{Materials and Methods}

\section{Panning of a Large Phage Displayed VH domain Library}

Recombinant human IGF-I and IGF-II (R\&D Systems) were used to pan a human VH domain phage library containing $10^{10}$ unique clones. Briefly, human IGF-II was biotinylated using EZ-Link Sulfo-NHS--Biotinylation Kit (Thermo Scientific) according to the instructions of the manufacturer. Biotinylated IGF-II was conjugated onto Dynabeads M-280 Streptavidin (Invitrogen) as target for the library panning. The phage clones were sequentially panned against IGF-I and IGF-II antigens.

\section{Mutagenesis by error-prone PCR}

Error-prone PCR of the entire antibody gene was performed using a GeneMorph ${ }^{\circledR}$ II Random Mutagenesis Kit according to the instructions of the manufacturer. Reaction products were purified by $1 \%$ agarose gel electrophoresis and concentrated by ultrafiltration with water.

\section{Yeast display selection}

Procedures for yeast library construction and isolation of high-affinity mutants were as previously described with minor modifications [27]. Before fluorescence-activated cell sorting (FACS), the yeast cells $\left(1 \times 10^{9}\right.$ cells $)$ were pre-selected with $10 \mu \mathrm{g}$ of IGF-II-conjugated magnetic beAds, followed by magnetic separation. Isolated yeast cells were cultured in $10 \mathrm{ml}$ of SDCAA media and induced in SG/RCAA media. Under stringent condition, yeast cells were sorted at $1,0.3$ and $0.1 \mu \mathrm{g} / \mathrm{ml}$ biotinylated-IGF-II. Yeast cells with high binding signals $(0.1-0.3 \%)$ were collected from sorting gates. After growth on SDCAA plates, yeast colonies were picked and sequenced.

\section{Antibody expression and purification}

The soluble eAds were expressed and purified as previously described [28]. The proteins were purified by Ni-NTA column. Eluted proteins were purified by the size-exclusion column (GE HiLoad 16/600 Superdex 75pg) using AKTA Avant 150 (GE Healthcare). A $4 \mathrm{ml}$ sample was loaded into the size-exclusion column and equilibrated with equilibrating buffer ( $1 \mathrm{mM}$ KH2PO4, $155 \mathrm{mM}$ Nacl, $3 \mathrm{mM}$ Na2HPO4-7H2O, PH 7.4). The column was washed continuously by using the buffer $(1 \mathrm{mM}$ 
KH2PO4, 155mM Nacl, 3mM Na2HPO4-7H2O, PH 7.4) at the flow rate of $1 \mathrm{ml} / \mathrm{min}$, and the fraction of the major area was collected.

\section{ELISA assay}

$25 \mathrm{ng}$ of IGF-I or IGF-II (Peprotech) per well were coated on 96-well ELISA plates overnight at $4^{\circ} \mathrm{C}$. Serial dilutions of eAds were incubated with antigens for $1 \mathrm{~h}$ at RT. Bound antibodies were detected with mouse anti-FLAG-HRP antibody (1:5,000, SigmaAldrich). The TMB substrates (Sigma-Aldrich) were added and the absorbance was read at $450 \mathrm{~nm}$.

\section{Competition and sandwich ELISA}

For sandwich ELISA, $100 \mathrm{ng}$ of anti-IGF-II IgG m610 per well were coated on ELISA plates. After blocking with PBS (3\% non-fat milk), 100 ng of IGF-II was added per well and captured by coated IgG m610. Serially diluted s7g1 and scFv m708.5 were added to wells and incubated for $1 \mathrm{~h}$. Bound antibodies were detected by HRP-conjugated anti-Flag antibody. The reaction was read at $450 \mathrm{~nm}$.

For inhibition of IGF-I or IGF-II binding to IGF-1R in ELISA, $50 \mathrm{ng}$ IGF-1R was directly coated on the ELISA plate. Serial dilutions of biotinylated IGF-I or IGF-II were added to ELISA plates in the presence of competitors (s7g1). Bound IGF-I or IGF-II was detected by HRP-conjugated streptavidin antibody. The reaction was read at $450 \mathrm{~nm}$. Shown are data with mean \pm SEM calculated from 3 separate experiments.

\section{Affinity measurements}

Binding affinities of the eAds to IGF-I/II were measured using surface plasmon resonance (SPR) by the Biacore T200 (GE Healthcare). The Procedures of Biacore were as previously described with some modifications [29]. Ligands, IGF-I or IGF-II, were immobilized onto the CM5 sensor chips through amine coupling method. A blank uncoated channel was used as reference during the injections. For analysis of the kinetics of interactions, different concentrations of EAds (5 - $200 \mathrm{nM}$ ) in running buffer (10 mM HEPES, $150 \mathrm{mM}$ NaCL, 3 mM EDTA, 0.05\% surfactant P-20, pH7.4) were flowed on the sensor chips at a flow rate of $30 \mu \mathrm{l} / \mathrm{min}$. The sensor ships were regenerated with glycine $(\mathrm{pH}$ 1.5) between each binding cycle. The association and dissociation phase data were fitted simultaneously to a 1:1 model by using BIAevaluation 3.1. All the experiments were performed at $25^{\circ} \mathrm{C}$.

\section{Competition flow cytometric assay}

Antibodies were mixed with $5 \mathrm{nmol} / \mathrm{L}$ biotinylated IGF-I or $1 \mathrm{nmol} / \mathrm{L}$ human biotinylated IGF-II (Peprotech) for $30 \mathrm{~min}$. The mixtures were incubated with $1 \times 10^{6}$ of MCF-7 cells in $100 \mu \mathrm{l}$ serum-free DMEM medium for $30 \mathrm{~min}$ on ice. After washing once, cells were stained with streptavidin-PE (1:50, Invitrogen) for $30 \mathrm{~min}$ on ice. The detection of bound IGFs was performed using a BD Bioscience FACScalibur. An irrelevant anti-HIV eAd m36 was used as the negative control.

\section{Phosphorylation assay}

The procedures of the IGF1R and IR phosphorylation assay were measured as previously described [18].

\section{Cell proliferation assay}

The procedures of the cell proliferation assay were performed as previously described [16]. Statistical significance was determined using by $\mathrm{t}$ test of Graphpad Prism software.

\section{Results}

\section{Selection of IGF-I and IGF-II cross-reactive human eAds}

Previously, a VH binder against a HIV-1 gp140 was isolated from a naive human antibody phage library. The $\mathrm{VH}$ sequence is closed to human germline (VH3-23) sequence. Based on the VH scaffold, a large $\left(2.5 \times 10^{10}\right)$ phage-displayed library was constructed by randomly mutating CDR1, and grafting CDR2s and CDR3s of heavy chains from five Fab libraries [30]. Here, we identified the human eAds against human IGFs by panning and screening one phagedisplayed $\mathrm{VH}$ domain library. Biotinylated human IGF-II was used as the antigen for panning. As a result, several human $\mathrm{VH}$ domain antibodies were isolated. Two dominant clones were identified to bind IGF-II in ELISA. One of these clones, 2g2, exhibited cross-reactivity for IGF-I and IGF-II.

\section{Affinity maturation of the eAd by random mutagenesis and yeast display}

To improve the affinity, random mutations were introduced into the $2 \mathrm{~g} 2$ gene by random mutagenesis PCR. The desired mutation rate was controlled by varying the initial amount of target DNA in the reaction or the number of amplification cycles performed. After sequencing, the low $(<9 / 1000 \mathrm{bp})$ mutation rate was achieved. The 2-7 nucleotide changes led to 1-4 amino acid substitutions per antibody. In protein evolution studies, low mutation frequencies are commonly employed to avoid the dramatic changing of structure. $30-40 \%$ of inserts were expressed in a mutant yeast library $\left(1 \times 10^{9}\right.$ size $)$ as verified by flow cytometry (not shown). The clones that did not display inserts were eliminated from the library with a subtraction using biotinylated IGF-II-conjugated beads. After the subtracted library 
was sequentially sorted three times for binding IGF-II under stringent conditions, a cell population with increased binding to IGF-II were enriched (Fig. 1A). Plasmids were isolated from the highest affinity clones from the final round of maturation. Sequence data of strong binders showed a recurrent mutant s7g1. Compared with 2g2, s7g1 had two amino acid substitutions in the heavy chain CDRs and two amino acid substitutions in the framework regions (Table 1).

Table 1. Mutations of s7gl amino acid sequences

\begin{tabular}{|c|c|c|}
\hline & FR1 & CDR2 \\
\hline 2g2 & QVQLVQSGGGLVQPGGSLRLSCAVSYYSLQ & ISGSGGST \\
\hline s7g1 & QVQLVQSGGGLVQPGGSLRLSCAVSYNSLQ & ISGSGGTT \\
\hline & FR3 & CDR3 \\
\hline $\mathbf{2 g 2}$ & YYADSVKGRFTISRDNSKNTLYLQMNTLRAEDTAMYYC & ARIRWLQDLDY \\
\hline s7g1 & LYADSVKGRFTISRDNSKNTLYLQMNTLRAEDTAMYYC & ARIRWLHDLDY \\
\hline
\end{tabular}

\section{Binding properties of affinity-maturated antibodies}

The s7g1 protein was expressed and purified. The purity was analyzed by the size-exclusion column (Fig. 1B) and SDS-PAGE (Fig. 1C) and. The binding ability was tested for IGF-I and IGF-II binding by
ELISA. It showed much more effective binding than the parental 2g2 (Fig. 2A). These substitutions resulted in a remarkable improvement of affinity while retaining its specificity, showing no crossreactivity with irrelevant antigens (Fig. 2B). Importantly, s7g1 did not bind to insulin. This means that s7g1 does not interfere on normal insulin metabolism.

The binding affinities were determined by the SPR method. With antigens coated onto biosensors, the values of affinities were determined. The obtained results are shown in Table 2. The improved s7g1 bound to IGF-I with a $\mathrm{K}_{\mathrm{D}}$ of $17.4 \mathrm{nM}$ and to IGF-II with a $K_{D}$ of $18.4 \mathrm{nM}$, whereas the values for $2 \mathrm{~g} 2$ were $120 \mathrm{nM}$ for IGF-I and $96 \mathrm{nM}$ for IGF-II. The affinity of s7g1 for IGF-I was increased 8-fold, whereas its affinity for IGF-II was increased 6-fold.

Table 2. Affinity comparison of EAds by surface plasmon resonance.

\begin{tabular}{lllll}
\hline & Antigen & Ka (1/Ms) & Kd (1/s) & KD (M) \\
\hline $\mathbf{2 g} \mathbf{2}$ & IGF-I & $7.90 \mathrm{E}+03$ & $1.11 \mathrm{E}-03$ & $1.41 \mathrm{E}-07$ \\
& IGF-II & $1.04 \mathrm{E}+04$ & $1.19 \mathrm{E}-03$ & $1.15 \mathrm{E}-07$ \\
$\mathbf{s 7 g 1}$ & IGF-I & $2.83 \mathrm{E}+04$ & $4.91 \mathrm{E}-04$ & $1.74 \mathrm{E}-08$ \\
& IGF-II & $3.02 \mathrm{E}+04$ & $5.65 \mathrm{E}-04$ & $1.87 \mathrm{E}-08$ \\
\hline
\end{tabular}

(A)

IGF-II: $10 \mathrm{nmol} / \mathrm{L}$

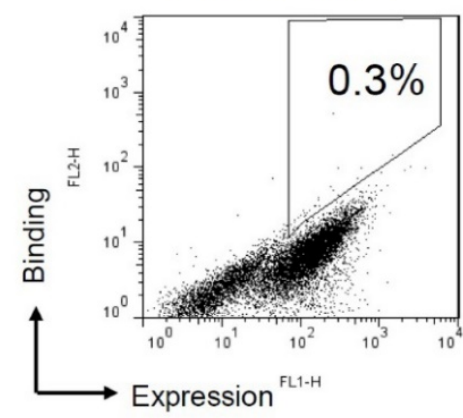

(B)

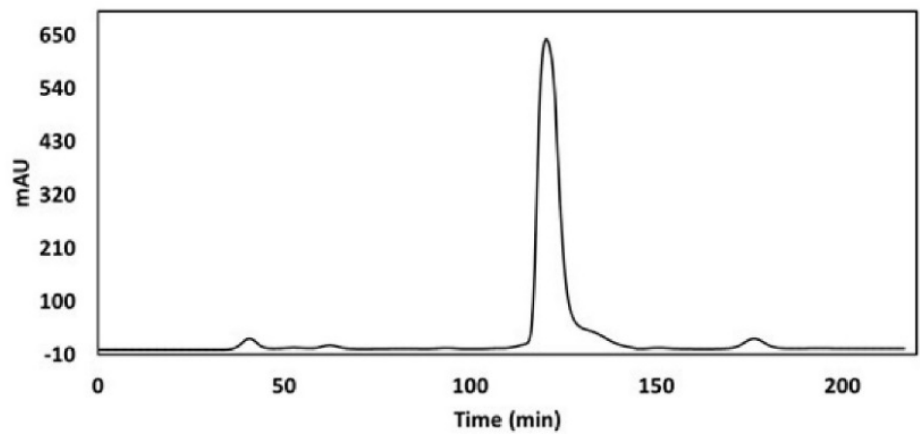

IGF-II: $5 \mathrm{nmol} / \mathrm{L}$

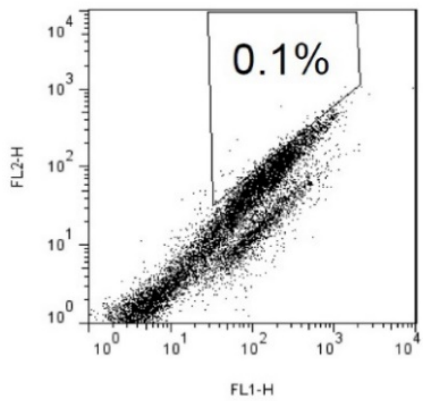

(C)

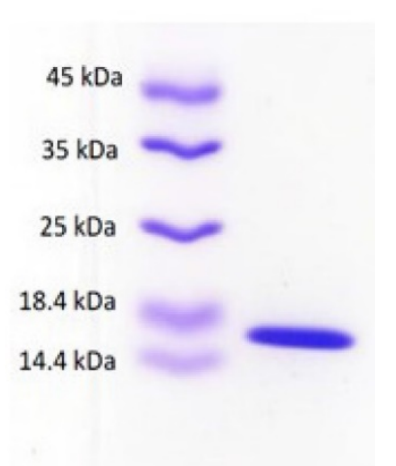

Figure 1. Affinity-maturation of the eAd by random mutagenesis and yeast display selection. (A) FACS selection of yeast-displayed $2 \mathrm{~g} 2 \mathrm{mutants}$. During three selections, yeast cells were stained with concentrations of biotinylated IGF-II at $10 \mathrm{nmol} / \mathrm{L}$ (sort1), $5 \mathrm{nmol} / \mathrm{L}$ (sort2) and $2.5 \mathrm{nmol} / \mathrm{L}$ (sort3), respectively. IGF-II-binding was detected by streptavidin-PE. Antibody expression on yeast was shown with mouse anti-c-myc antibody followed by FITC goat anti-mouse secondary antibody. The $0.1-0.3 \%$ cells were selected from sort gates. (B) Purification of soluble s7g1 by the size-exclusion column. (C) SDS-PAGE analysis of purified s7g. 
(A)

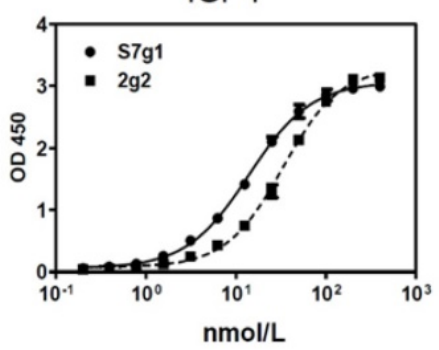

IGF-II

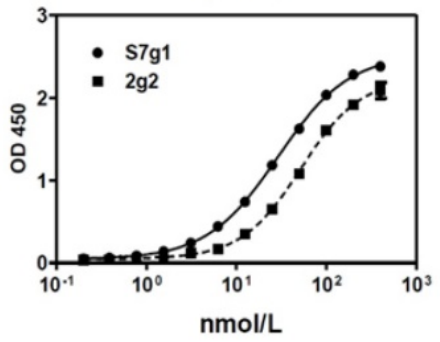

(B)

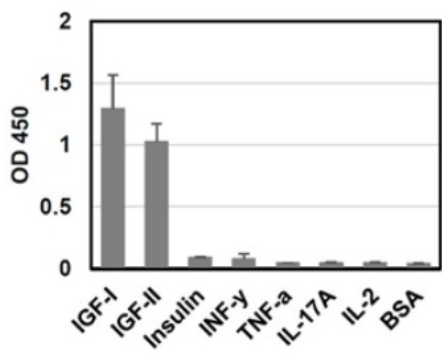

Figure 2. ELISA analysis. (A) Binding of $2 \mathrm{~g} 2$ and s7gl against IGF-I and IGF-II. (B) Specificity of s7gl against different antigens. Purified eAds (10nmol/L) were used to detect coated antigens, followed by detected with a HRP-conjugated anti-FLAG antibody. Shown are data with mean \pm SEM calculated from 3 separate experiments.

(A)

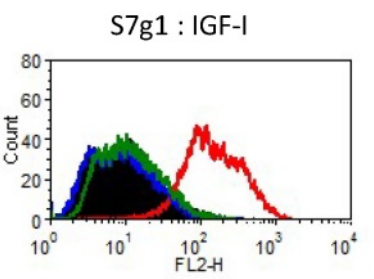

S7g1 : IGF-II

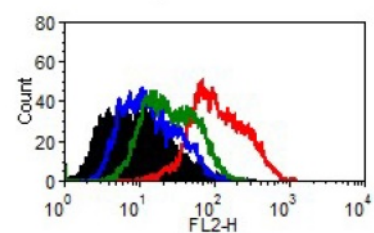

$2 \mathrm{~g} 2:$ IGF-I

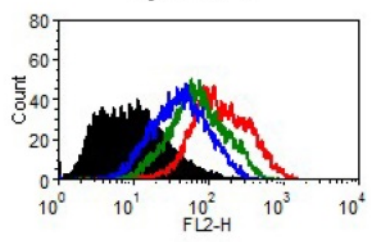

2g2 : IGF-II

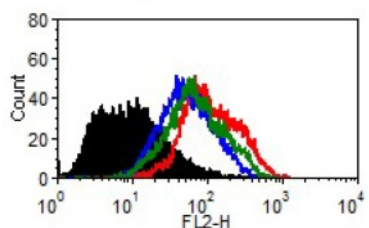

(B)
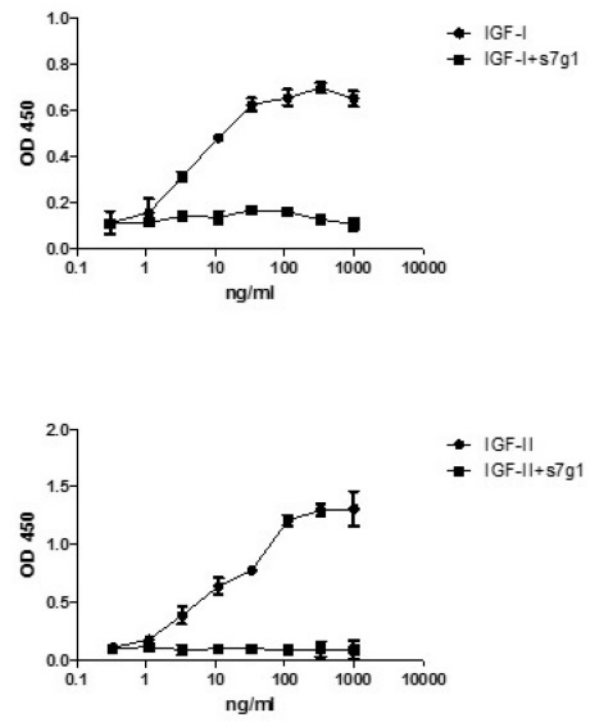

Figure 3. Blockade of IGF-I and IGF-II binding to receptors by eAds. (A) Inhibition of IGF-I or IGF-II binding to MCF-7 cells. Biotinylated IGF-I was preincubated with s7gl and control m36 at concentrations of $10 \mathrm{nmol} / \mathrm{L}$ or $100 \mathrm{nmol} / \mathrm{L}$. Then, mixtures were used to stain with MCF-7 cells. Cells were detected by streptavidin-PE. In the graphs, black lines are for cells without staining. Green and blue lines are for tested antibodies at $10 \mathrm{nmol} / \mathrm{L}$ and $100 \mathrm{nmol} / \mathrm{L}$, separately. Red lines represent binding of IGF-I or IGF-II alone. (B) Inhibition of IGF-I or IGF-II binding to IGF-1R in ELISA. IGF-1R was directly coated on the ELISA plate. Serial dilutions of biotinylated IGF-I or IGF-II were added to ELISA plates in the presence of competitors (s7gI). Bound IGF-I or IGF-II was detected by HRP-conjugated streptavidin antibody. The reaction was read at $450 \mathrm{~nm}$. Shown are data with mean \pm SEM calculated from 3 separate experiments.

(A)

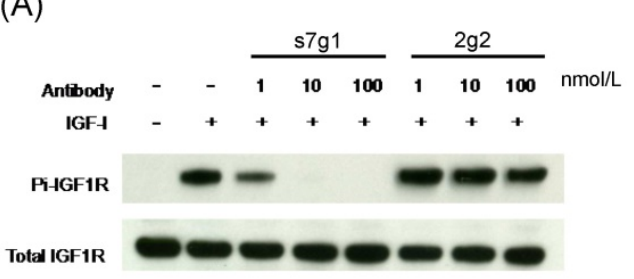

(C)

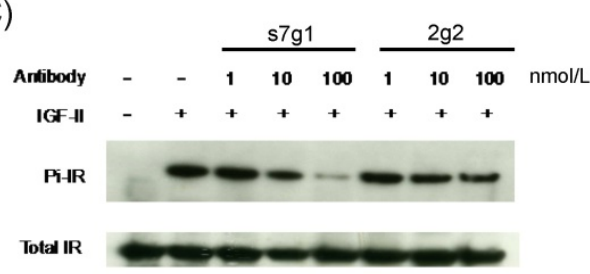

(B)

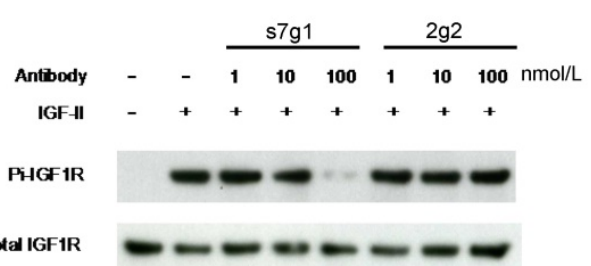

Figure 4. Inhibition of IGF-IR and IR phosphorylation in MCF-7 cells. MCF-7 cells were starved in serum free medium for 5 h, followed by addition of treatment medium with $1 \mathrm{nmol} / \mathrm{L}$ IGF-I or $5 \mathrm{nmol} / \mathrm{L}$ IGF-II with varying concentrations of s7g1 or $2 \mathrm{~g} 2$. After cells were lysed, target proteins were immunoprecipitated by specific antibody conjugated resins. (A-B) Phosphorylation of IGF-IR induced by IGF-I or IGF-II was detected with a phosphor-tyrosine specific antibody. (C) Phosphorylation of IR induced by IGF-II was detected as following above methods. The total amount of IGF-1R or IR was detected by the same polyclonal antibody used for the immuneprecipitation. Data showed a representative from 3 separate experiments. 


\section{Blockade of IGF-I/IGF-II binding and receptor phosphorylation}

Signaling pathways of IGF-IR and IR are activated by binding of IGFs. To determine whether s7g1 inhibits binding of IGF-I and IGF-II to their receptors, we studied IGF-IR and IR signaling using the breast cancer cell line MCF-7, which is known to express high levels of both receptors. S7g1 exhibited inhibition in a flow cytometry-based assay. As shown in Fig. 3A, the binding of biotinylated IGF-I or IGF-II to their receptors on MCF-7 cells was completely blocked. The control eAd m36 seemed to slightly affect the IGF-I/II binding due to their same scaffold. In a competition ELISA, the binding of IGF-I or IGF-II to IGF-1R was also competed by s7g1 (Fig. 3B).

(A)

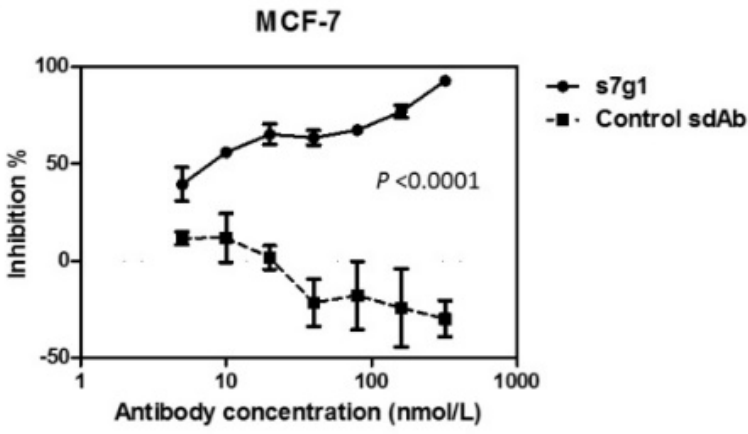

(B)

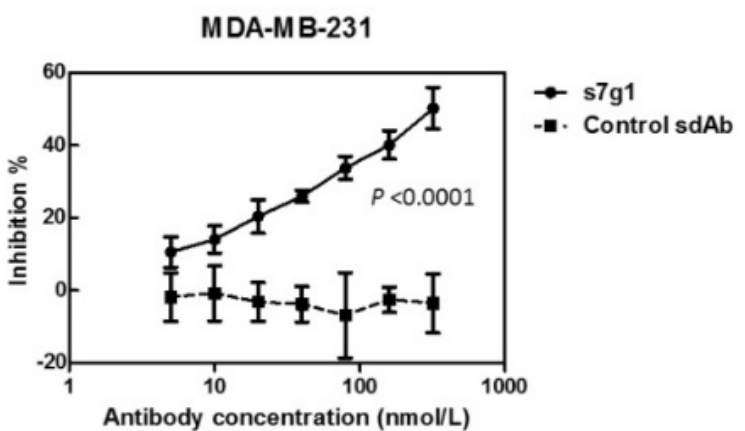

Figure 5 Growth inhibition of breast cancer cells. Different concentrations of s7g1 and control $\mathrm{m} 36$ were preincubated with IGF-I and IGF-II. Then, breast cancer cell lines MCF-7 (A) and MDA-MB-231 (B) were cultured in the serum-free media in the presence of the mixture of antibody and IGFs. After 3 days, numbers of viable cells were determined using MTS method. Positive control were cells in serum-free medium with IGFs. Blank control was cells in serum-free medium without any IGFs. Shown are data with mean \pm SEM calculated from 3 separate experiments.

Meanwhile, s7g1 also inhibited the transmembrane signaling mediated by IGF-IR and IR in MCF-7 cells. Median serum concentrations of free IGF-I or IGF-II in individuals with breast lesions is approximately $0.7 \mathrm{nmol} / \mathrm{L}$ or $1.8 \mathrm{nmol} / \mathrm{L}$ [31]. The concentrations of $1 \mathrm{nmol} / \mathrm{L}$ of IGF-I and $5 \mathrm{nmol} / \mathrm{L}$ of IGF-II around serum concentrations were used in the experiments. Immunoblottings showed that s7g1 inhibited IGF-I-induced or IGF-II-induced receptor phosphorylation (Fig. 4A and B). It was capable of inhibiting IGF-I-induced phosphorylation of IGF1R at $100 \mathrm{nmol} / \mathrm{L}$. In addition, the IGF-II-induced phosphorylation of IGF1R was inhibited by s7g1 at concentrations between $10 \mathrm{nmol} / \mathrm{L}$ and $100 \mathrm{nmol} / \mathrm{L}$. $2 \mathrm{~g} 2$ showed no obvious inhibitory activity at the same concentration. As shown in Fig. 4C, s7g1 showed strong but incomplete inhibition of $p$-IR at 100 nmol/L. Inhibition was also observed when the antibody concentration was decreased to $10 \mathrm{nmol} / \mathrm{L}$. 2g2 seems to slightly inhibit IR phosphorylation.

\section{Inhibition of tumor cell growth}

The activity of s7g1 was tested in a cell growth assay using two breast cancer cell lines MCF-7 and MDA-MB-231. After 3 days of treatment, s7g1 exhibited the inhibitory ability. The cell growth of breast cancer cells was remarkably inhibited at the concentrations ranging from $5 \mathrm{nmol} / \mathrm{L}$ to $320 \mathrm{nmol} / \mathrm{L}$ (Fig. 5). Even at a concentration of $5 \mathrm{nmol} / \mathrm{L} \mathrm{s7g1,}$ $40 \%$ inhibition of MCF-7 cells was even achieved. By comparison, the control m36 did not show measurable inhibition of cell growth. The data revealed that s7g1 effectively inhibited breast cancer cells growth in vitro.

\section{Epitope analysis}

The competition ELISA showed that s7g1 competed with m708.5 in binding to IGF-I and IGF-II (Fig. 6A and B). However, they showed different binding to m610-captured IGF-II in sandwich ELISA (Fig. 6C). This means that the binding epitopes of the two antibodies are distinct, but overlapped on IGFs.
(A)

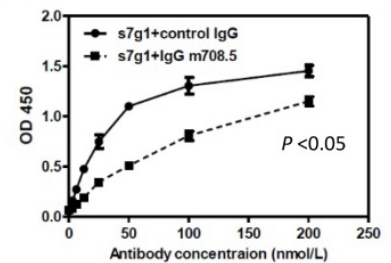

(C)

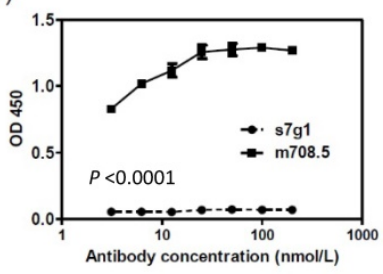

(B)

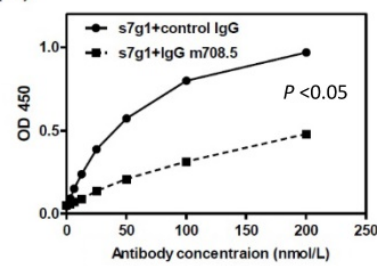

Figure 6. Competition of s7g1 with m708.5 binding to antigens. (A-B) competition ELISA. IGF-I (A) or IGF-II (B) was directly coated on the ELISA plate. Serial dilutions of $s 7 \mathrm{gl}$ were added in the presence of competitors (lgG m708.5 or a control $\operatorname{lgG}$ ). Bound $\mathrm{s} 7 \mathrm{gl}$ was detected by HRP-conjugated anti-Flag antibody. (C) Sandwich ELISA. IGF-II was captured with IgG m610 coated on the ELISA plate. Bound s7g1 and scFv m708.5 were detected by HRP-conjugated anti-FLAG antibody. Shown are data with mean \pm SEM calculated from 3 separate experiments. 


\section{Discussion}

The IGF signaling pathway is important in breast cancer [32]. Increased expression of IGF-1R, IGF-I, and IGF-II has been observed in breast cancer. Aberrant IGF-I and IGF-II contribute to disease aggressiveness through autocrine, paracrine and endocrine interactions. It is believed that IR activated by IGF-II contributes to resistance to anti-IGF-1R therapy [16]. IR is a predominant IGF signaling receptor in breast cancer [6], suggesting that the blockade of IGF signaling through IGF-1R and IR may be an effective treatment strategy. Previously, we generated fully human monoclonal antibodies m610 and m708.5 [18] as well as bispecific antibodies m67 and m660 [14, 15] that inhibited both IGF-1R- and IR-mediated signal through neutralizing IGF-I and/or IGF-II. Anti-IGF mAbs and bsAbs are clearly effective to reduce plasma IGF-I and IGF-II due to their high affinity (pM) and relatively long half-lives. The monoclonal antibody m708.5 has shown satisfying preclinical activities against a broad spectrum of human cell lines. The combination of m708.5 and mTOR inhibitors significantly decreased neuroblastoma growth in vitro and in vivo.

One anti-HIV-1 eAd m36 was previously derived from the human $\mathrm{VH}$ domain scaffold. This eAd is clinically used in bispecific antibodies through fusing $\mathrm{m} 36$ on the CD4-specific ibalizumab for treating HIV-1 infection [33, 34]. Here, we further developed one eAd s7g1 (15 kDa) that cross-reacts with IGF-I and IGF-II. s7g1 is the first eAd that can cross-neutralize human IGF-I/II. To decrease IGF transduction, serum and tissue levels of ligands (IGF-I and IGF-II) need to be continuously neutralized. Therefore, high affinity of antibodies is required to fulfil neutralization of the continuous sequestration of the ligand from its receptor. More often, to achieve high affinity, the complementarity determining regions (CDRs) of antibodies are most likely to generate affinity maturation [35]. Our previous studies demonstrated that the mutation points located in both CDRs and framework regions were likely involved into antibody affinity maturation [36]. Therefore, random mutations were introduced into both CDR and the framework regions of s7g1. Consequently, the improvement in affinity was found to be 8- or 6-fold compared with the original clone. Affinity analysis shows s7g1 bound to IGF-I with a $K_{D}$ of $17 \mathrm{nM}$ and IGF-II with a KD of $18 \mathrm{nM}$. The affinity values of s7g1 to human IGF-I and IGF-II are similar probably due to $62 \%$ sequence identity of human IGF-I and IGF-II. The binding affinities of s7g1 are modest but typical of those eAds. Thought some small antibody domains showed very high affinities, they exhibited weak tumor penetration because the high affinity resulted in perivascular localization [37].

s7g1 is sufficient to block signal transduction mediated by IGF-I/II interacting with their receptors on tumor cells and inhibits breast tumor cell growth in vitro. Importantly, s7g1 suppresses IGF-II-mediated IR activation by neutralizing IGF-II. We are not sure about s7g1's exact epitopes on IGF-I/II. It might share epitopes as $\mathrm{m} 708.5$, but different epitopes on IGF-II with m610. It could be due to a smaller paratope of the small-size eAd compared to IgGs. Its epitope is unique and was not previously targeted by other antibodies. Compared with IgG-size antibodies, these small antibodies are more rapidly eliminated than full-size antibodies through the kidney [38]. These small eAds have distinct advantages because they potentially recognize epitopes in protein targets not accessible to large antibodies.

In conclusion, s7g1 offers a possible therapeutic candidate addition to other anti-IGF mAbs. We will consider to extend the circulating half-life of anti-IGF-I/II eAds through the use of protein engineering, such as polyethylene glycol (PEG) modification [39], and genetic protein fusion [40]. Due to its size of $15 \mathrm{kDa}$ and its general robustness, s7g1 is expected to extravagate and diffuse into tissue effectively. We believe that it facilitates the elimination of autocrine IGFs secreted from stroma within tumor microenvironment because small antibodies easily overcome tumor barriers. It will also be interesting to evaluate its in vivo bioactivity in combination with other anti-IGF-I/II mAbs, such as $\mathrm{m} 708.5$, and chemotherapy drugs.

\section{Acknowledgements}

This work was supported by the Science and Technology Development Fund of Macau (FDCT/ 131/2016/A3), the Guangdong Science and Technology Program (2016A050502034 and 2017B030301018), the Guangzhou Science and Technology Program (201807010004), Natural Science Foundation of Guangdong (2015A0 30313741), Shenzhen Science and Technology Innovation Committee (JCYJ20160531171744232, JCY J20150401145529036, JCYJ20160608140912962, ZDSYS 20140509142721429), Start-up Research Grand (SRG2 016-00082-FHS) and the intramural research program of Faculty of Health Sciences, University of Macau, and National Natural Science Foundation of China (31440041, 31670753, 31770996).

\section{Competing Interests}

The authors have declared that no competing interest exists. 


\section{References}

1. Samani AA, Yakar S, LeRoith D, Brodt P. The role of the IGF system in cancer growth and metastasis: overview and recent insights. Endocrine reviews. 2007; 28: $20-47$.

2. Christopoulos PF, Msaouel P, Koutsilieris M. The role of the insulin-like growth factor-1 system in breast cancer. Molecular cancer. 2015; 14: 43.

3. Pollak M. Insulin and insulin-like growth factor signalling in neoplasia. Nat Rev Cancer. 2008; 8: 915-28.

4. Brahmkhatri VP, Prasanna C, Atreya HS. Insulin-like growth factor system in cancer: novel targeted therapies. BioMed research international. 2015; 2015: 538019.

5. Malaguarnera R, Belfiore A. The insulin receptor: a new target for cancer therapy. Frontiers in endocrinology. 2011; 2: 93.

6. Huang J, Morehouse C, Streicher K, Higgs BW, Gao J, Czapiga M, et al. Altered expression of insulin receptor isoforms in breast cancer. PloS one. 2011; 6: e26177.

7. Feng Y, Dimitrov DS. Antibody-based therapeutics against components of the IGF system. Oncoimmunology. 2012; 1: 1390-1.

8. Rodon J, DeSantos V, Ferry RJ, Jr., Kurzrock R. Early drug development of inhibitors of the insulin-like growth factor-I receptor pathway: lessons from the first clinical trials. Molecular cancer therapeutics. 2008; 7: 2575-88.

9. Belfiore A, Malaguarnera R. Insulin receptor and cancer. Endocrine-related cancer. 2011; 18: R125-47.

10. Buck E, Gokhale PC, Koujak S, Brown E, Eyzaguirre A, Tao N, et al. Compensatory insulin receptor (IR) activation on inhibition of insulin-like growth factor-1 receptor (IGF-1R): rationale for cotargeting IGF-1R and IR in cancer. Molecular cancer therapeutics. 2010; 9: 2652-64.

11. Carboni JM, Wittman M, Yang Z, Lee F, Greer A, Hurlburt W, et al. BMS-754807, a small molecule inhibitor of insulin-like growth factor-1R/IR. Molecular cancer therapeutics. 2009; 8: 3341-9.

12. Yee D, Favoni RE, Lebovic GS, Lombana F, Powell DR, Reynolds CP, et al. Insulin-like growth factor I expression by tumors of neuroectodermal origin with the $t(11 ; 22)$ chromosomal translocation. A potential autocrine growth factor. The Journal of clinical investigation. 1990; 86: 1806-14.

13. Giani C, Pinchera A, Rasmussen A, Fierabracci P, Bonacci R, Campini D, et al. Stromal IGF-II messenger RNA in breast cancer: relationship with progesterone receptor expressed by malignant epithelial cells. Journal of endocrinological investigation. 1998; 21: 160-5.

14. Chen W, Feng $Y$, Zhao $Q$, Zhu Z, Dimitrov DS. Human monoclonal antibodies targeting nonoverlapping epitopes on insulin-like growth factor II as a novel type of candidate cancer therapeutics. Molecular cancer therapeutics. 2012; 11: 1400-10.

15. Feng $Y$, Zhao Q, Chen W, Wang Y, Crowder K, Dimitrov DS. A new bispecific antibody targeting non-overlapping epitopes on IGF2: design, in vitro characterization and pharmacokinetics in macaques. Experimental and molecular pathology. 2014; 97: 359-67.

16. Zhao Q, Tran H, Dimitrov DS, Cheung NK. A dual-specific anti-IGF-1/IGF-2 human monoclonal antibody alone and in combination with temsirolimus for therapy of neuroblastoma. International journal of cancer. 2015; 137: 2243-52.

17. Haluska P, Menefee M, Plimack ER, Rosenberg J, Northfelt D, LaVallee T, et al Phase I dose-escalation study of MEDI-573, a bispecific, antiligand monoclonal antibody against IGFI and IGFII, in patients with advanced solid tumors. Clinical cancer research : an official journal of the American Association for Cancer Research. 2014; 20: 4747-57.

18. Zhao Q, Feng Y, Zhu Z, Dimitrov DS. Human monoclonal antibody fragments binding to insulin-like growth factors I and II with picomolar affinity. Molecular cancer therapeutics. 2011; 10: 1677-85.

19. Feng Y, Zhu Z, Xiao X, Choudhry V, Barrett JC, Dimitrov DS. Novel human monoclonal antibodies to insulin-like growth factor (IGF)-II that potently inhibit the IGF receptor type I signal transduction function. Molecular cancer therapeutics. 2006; 5: 114-20.

20. Kimura T, Kuwata T, Ashimine S, Yamazaki M, Yamauchi C, Nagai K, et al. Targeting of bone-derived insulin-like growth factor-II by a human neutralizing antibody suppresses the growth of prostate cancer cells in a human bone environment. Clinical cancer research : an official journal of the American Association for Cancer Research. 2010; 16: 121-9.

21. Carter P. Improving the efficacy of antibody-based cancer therapies. Nature reviews Cancer. 2001; 1: 118-29.

22. Baker JH, Lindquist KE, Huxham LA, Kyle AH, Sy JT, Minchinton AI. Direct visualization of heterogeneous extravascular distribution of trastuzumab in human epidermal growth factor receptor type 2 overexpressing xenografts. Clinical cancer research : an official journal of the American Association for Cancer Research. 2008; 14: 2171-9.

23. Dimitrov DS. Engineered $\mathrm{CH} 2$ domains (nanoantibodies). mAbs. 2009; 1: 26-8.

24. Chen W, Feng Y, Gong R, Zhu Z, Wang Y, Zhao Q, et al. Engineered single human CD4 domains as potent HIV-1 inhibitors and components of vaccine immunogens. Journal of virology. 2011; 85: 9395-405.

25. Gong R, Wang Y, Feng Y, Zhao Q, Dimitrov DS. Shortened engineered human antibody $\mathrm{CH} 2$ domains: increased stability and binding to the human neonatal Fc receptor. The Journal of biological chemistry. 2011; 286: 27288-93.

26. Li D, Gong R, Zheng J, Chen X, Dimitrov DS, Zhao Q. Engineered antibody $\mathrm{CH} 2$ domains binding to nucleolin: Isolation, characterization and improvement of aggregation. Biochemical and biophysical research communications. 2017; 485: 446-53.
27. Zhao Q, Zhu Z, Dimitrov DS. Yeast display of engineered antibody domains. Methods in molecular biology. 2012; 899: 73-84.

28. Zhao Q, Chan YW, Lee SS, Cheung WT. One-step expression and purification of single-chain variable antibody fragment using an improved hexahistidine tag phagemid vector. Protein expression and purification. 2009; 68: 190-5.

29. Xu T, Ying T, Wang L, Zhang XD, Wang Y, Kang L, et al. A native-like bispecific antibody suppresses the inflammatory cytokine response by simultaneously neutralizing tumor necrosis factor-alpha and interleukin-17A. Oncotarget. 2017; 8: 81860-72

30. Chen W, Zhu Z, Feng Y, Xiao X, Dimitrov DS. Construction of a large phage-displayed human antibody domain library with a scaffold based on a newly identified highly soluble, stable heavy chain variable domain. Journal of molecular biology. 2008; 382: 779-89.

31. Singer CF, Mogg M, Koestler W, Pacher M, Marton E, Kubista E, et al. Insulin-like growth factor (IGF)-I and IGF-II serum concentrations in patients with benign and malignant breast lesions: free IGF-II is correlated with breast cancer size. Clinical cancer research : an official journal of the American Association for Cancer Research. 2004; 10: 4003-9.

32. Sachdev D, Yee D. The IGF system and breast cancer. Endocrine-related cancer. 2001; 8: 197-209.

33. Ferrari G, Haynes BF, Koenig S, Nordstrom JL, Margolis DM, Tomaras GD. Envelope-specific antibodies and antibody-derived molecules for treating and curing HIV infection. Nature reviews Drug discovery. 2016; 15: 823-34.

34. Chen W, Zhu Z, Feng Y, Dimitrov DS. Human domain antibodies to conserved sterically restricted regions on gp120 as exceptionally potent cross-reactive HIV-1 neutralizers. Proceedings of the National Academy of Sciences of the United States of America. 2008; 105: 17121-6.

35. Tiller KE, Li L, Kumar S, Julian MC, Garde S, Tessier PM. Arginine mutations in antibody complementarity-determining regions display context-dependent affinity/specificity trade-offs. The Journal of biological chemistry. 2017.

36. Ahmed $\mathrm{M}$, Cheng $\mathrm{M}$, Zhao $\mathrm{O}$, Goldgur $\mathrm{Y}$, Cheal $\mathrm{SM}$, Guo HF, et al. Humanized Affinity-matured Monoclonal Antibody 8H9 Has Potent Antitumor Activity and Binds to FG Loop of Tumor Antigen B7-H3. The Journal of biological chemistry. 2015; 290: 30018-29.

37. Vaneycken I, Devoogdt N, Van Gassen N, Vincke C, Xavier C, Wernery U, et al. Preclinical screening of anti-HER2 nanobodies for molecular imaging of breast cancer. FASEB journal : official publication of the Federation of American Societies for Experimental Biology. 2011; 25: 2433-46.

38. Vazquez-Lombardi R, Phan TG, Zimmermann C, Lowe D, Jermutus L, Christ D. Challenges and opportunities for non-antibody scaffold drugs. Drug discovery today. 2015; 20: 1271-83.

39. Stork R, Campigna E, Robert B, Muller D, Kontermann RE. Biodistribution of a bispecific single-chain diabody and its half-life extended derivatives. The Journal of biological chemistry. 2009; 284: 25612-9.

40. Hutt M, Farber-Schwarz A, Unverdorben F, Richter F, Kontermann RE. Plasma half-life extension of small recombinant antibodies by fusion to immunoglobulin-binding domains. The Journal of biological chemistry. 2012; 287: 4462-9. 